\title{
Coupled Reactors Analysis: New Needs and Advances Using Monte Carlo Methodology
}

\author{
M. Aufiero ${ }^{1}$, G. Palmiotti ${ }^{2,3}$, M. Salvatores ${ }^{2}$, S. Sen ${ }^{2}$ \\ ${ }^{1}$ University of California, Berkeley, Department of Nuclear Engineering, Berkeley, CA 94720-1730 USA \\ ${ }^{2}$ NSDA Division, Idaho National Laboratory, P.O. Box 1625, Idaho Falls, ID 83415-3860, USA \\ ${ }^{3}$ Corresponding author: Giuseppe.Palmiotti@inl.gov
}

\begin{abstract}
Coupled reactors and the coupling features of large or heterogeneous core reactors can be investigated with the Avery's theory; however, the complex geometries that are often encountered in association with coupled reactors, require a detailed geometry description that can be easily provided by modern Monte Carlo (MC) codes. The results presented in this paper show that the MC code SERPENT has been successfully modified in order to compute the needed quantities like coupling coefficients. Moreover, the capability for calculating sensitivities to the quantities of interest for coupling reactors has been developed and implemented in SERPENT.
\end{abstract}

Keywords: Reactor design; Monte Carlo; Coupled reactors; Sensitivity analysis.

\section{1- Introduction}

The idea of "coupled" reactors, i.e. reactors with e.g. two core regions with different spectra, neutronically coupled with or w/o a geometrical barrier (intermediate zone or buffer, more often without fissile material), has been originally proposed by R. Avery (Avery, 1958). The motivation at the time was to couple a fast and a thermal assembly in order to obtain a combined system that can have the breeding ratio characteristics of an all-fast spectrum system and at the same time exhibiting a prompt neutron lifetime characteristic of a thermal neutron system, i.e. much higher than the one for an all-fast neutron system, considered at the time as a potential drawback.

The principle was experimentally tested (Toppel, 1957). Successively the fast-thermal coupling was often used to build fast neutron experimental facilities with a limited all-fast neutron zone (see for example Meister et al., 1964, and 4 Bustraan et al., 1970); moreover that type of system was also experimentally realized e.g. in order to test the principle of specific coupled systems, as the pulse coupled reactor system consisting of fast burst reactor and a subcritical thermal module (Kukharchuk et al., 2000) or even a cluster of rocket reactors (Seale, 1964).

More recently, the potential of a coupled Fast-thermal reactor as versatile test reactor has been pointed out (Sen et al., 2016).

Theoretical methods have been developed to describe these systems both in steady and in transient conditions (see e.g. Avery, 1958, Baldwin, 1959, Komata, 1969, and Abramov, 2001).

The "decoupling" of spatial regions in a reactor, is a well-recognized phenomenon that has been pointed out the early reactor physics studies. The decoupling/coupling effects are 
physics effects that can be found not only in the type of coupled systems mentioned above, but also in large reactors, where spatial regions can act as regions weakly or more strongly coupled. The potential flux tiltiness in the system can be associated for example to the socalled Boltzmann operator eigenvalue separation (see e.g. Abramov, 2001, ) or, in case of a coupled system, the "flux tilting" between e.g. two regions, associated to the flux ratio in these regions, can be directly related to the ratio of the sub-criticality in the two regions (Seale, 1964).

In all cases, the performance analysis of these systems, both in steady or in transient conditions, requires a deep understanding of the, sometime non-conventional, physics phenomena, from the appropriate preparation of cross-sections to the evaluation of the impact of uncertainties and e.g. of the dependence of the specific coupling phenomena from nuclear data uncertainties.

Moreover, the detailed description of the geometry of typical coupled systems requires an appropriate Monte Carlo treatment; see e.g. Fig.1 from (Sen et al, 2016):

Figure 1. XY view of a coupled thermal-fast irradiation test reactor (VCTR from Sen et al, 2016)

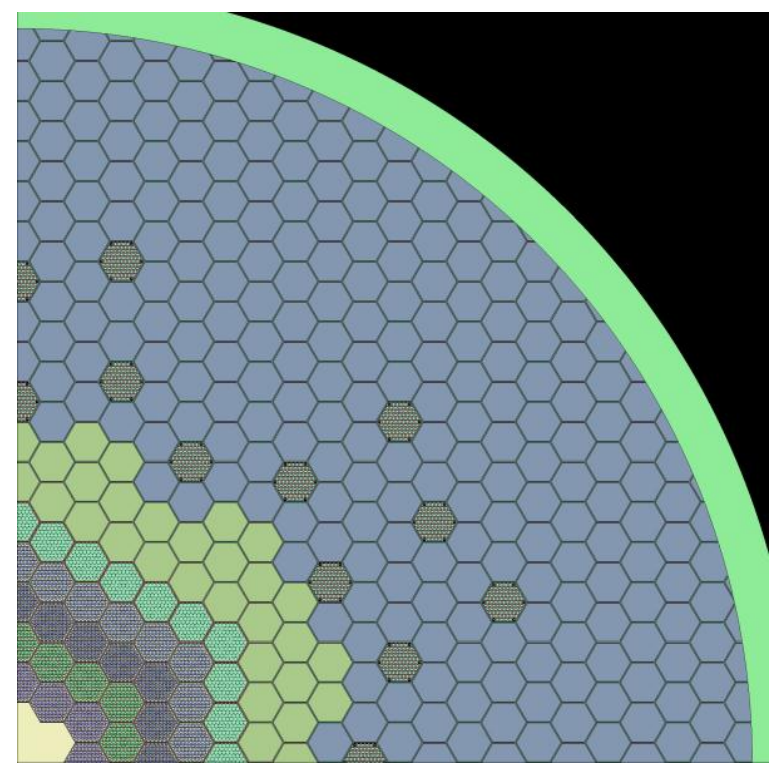

The need of using a Monte Carlo code implies its extension to the evaluation of the typical coupling parameters (see next section) and the development of a full sensitivity analysis capability. This capability should go beyond the standard reactor integral parameter sensitivity, but should be extended to coupling features of the system, kinetic parameters and spatial power distributions.

In this paper we will describe the theoretical formulations that have been developed for this purpose and their practical implementation together with some significant applications. 


\section{2- Short summary of some specific features of the Avery's theory}

For the purpose of this paper, we will remind shortly the simplest form of the coupled reactor reactivity and criticality conditions that are given by (in the case of two coupled "regions", (see Avery, 1958)) for the coupling coefficients $k_{i j}$ :

$$
\begin{gathered}
\left|\begin{array}{cc}
\left.k_{11}-1\right) & k_{12} \\
k_{21} & \left(k_{22}-1\right)
\end{array}\right|=0, \\
k_{12} k_{21}=\Delta_{1} \Delta_{2} .
\end{gathered}
$$

where

$$
\Delta_{j}=1-k_{j j}
$$

If we indicate with $S_{i}$ the power of region $i$, the power ratio in the two coupled regions is given by:

$$
\frac{S_{1}}{S_{2}}=\frac{k_{12}}{\Delta_{1}}=\frac{\Delta_{2}}{k_{21}}
$$

In the general case of $N$ regions one has to solve the following system:

$$
\left(\begin{array}{c}
k_{11} k_{12} \ldots k_{1 N} \\
k_{21} k_{22} \ldots k_{2 N} \\
\ldots \\
\ldots \\
\ldots \\
k_{N 1} k_{N 2} \ldots k_{N N}
\end{array}\right)\left(\begin{array}{c}
S_{1} \\
S_{2} \\
\cdot \\
\cdot \\
\cdot \\
S_{N}
\end{array}\right)=k\left(\begin{array}{c}
S_{1} \\
S_{2} \\
\cdot \\
\cdot \\
\cdot \\
S_{N}
\end{array}\right)
$$

The reactivity $\rho$ is defined as:

$$
\rho \approx \sum_{i=1}^{N} \propto_{i} \frac{\delta v_{i}}{v_{i}}
$$

where

$$
\propto_{i}=\frac{\frac{1}{\Delta_{i}}}{\sum_{j=1}^{N} \frac{1}{\Delta_{j}}}
$$


The point kinetics equations are given (using exactly the same notations as in Avery, 1958) by:

$$
\begin{gathered}
l_{j k} \frac{d S_{j k}}{d t}=k_{j k}(1-\beta) \sum_{m=1}^{N} S_{k m}-S_{j k}+k_{j k} \sum_{i=1}^{D} \lambda_{i} C_{k i} \\
\frac{d C_{k i}}{d t}=\beta_{i} \sum_{m=1}^{N} S_{k m}-\lambda_{i} C_{k i} .
\end{gathered}
$$

Where $D$ is the number of delayed neutron families.

The power in region $k$ is given by:

$$
S_{k}=\sum_{m=1}^{N} S_{k m}
$$

In practice, the following expressions are the solution of the standard Boltzmann equations and that account for the space and energy dependence:

$$
\begin{gathered}
S_{j}=\int\left[v \sigma_{f}(r, v)\right]_{j} \varphi(r, v) d r d v . \\
S_{j k}=\frac{\int\left[v \sigma_{f}(r, v)\right]_{j} \varphi(r, v) d r d v \times \int \chi\left(v^{\prime}\right) \varphi^{*}\left(r, v^{\prime}\right)\left[v \sigma_{f}(r, v)\right]_{j} \varphi_{k}(r, v) d r d v d v^{\prime}}{\int \chi\left(v^{\prime}\right) \varphi^{*}\left(r, v^{\prime}\right)\left[v \sigma_{f}(r, v)\right]_{j} \varphi(r, v) d r d v d v^{\prime}} \\
k_{j k}=\frac{S_{j k}}{S_{k}} \\
k_{j k}=\frac{\int\left[v \sigma_{f}(r, v)\right]_{j} \varphi(r, v) d r, d v}{\int\left[v \sigma_{f}(r, v)\right]_{k} \varphi(r, v) d r d v} \times \frac{\int \chi\left(v^{\prime}\right) \varphi^{*}\left(r, v^{\prime}\right)\left[v \sigma_{f}(r, v)\right]_{j} \varphi_{k}(r, v) d r d v d v^{\prime}}{\int \chi\left(v^{\prime}\right) \varphi^{*}\left(r, v^{\prime}\right)\left[v \sigma_{f}(r, v)\right]_{j} \varphi(r, v) d r d v d v^{\prime}} .
\end{gathered}
$$

Using these expressions, one can solve the kinetics equations given above accounting for the energy and the within-region space dependence.

It has been shown the overall Avery formulation as indicated above, is well suited for a rather quick analysis not only of coupled systems of the type shown in fig. 1, but also for the analysis of a wider class of systems of current interest. This is the case of the coupling of different regions in large, potentially decoupled systems like the proposed axially 
heterogeneous fast reactor ASTRID (Varaine et al., 2012), as shown in a simplified model below (Figure 2), where the fertile region in the middle of the core can induce weak coupling effects among the different core regions:

In fact the $k_{i j}$ coefficients give a rather accurate description of the coupling mechanism between core regions and the power evolution by region gives a first quantitative indication of potential power "tiltiness" e.g. in case of asymmetrical reactivity insertion (Palmiotti, Salvatores at al., 2016)

Figure 2. ASTRID simplified model.

\begin{tabular}{|c|c|}
\hline Upper core & \\
\hline Fertile & \\
\hline Lower core & \\
\hline & \\
\hline
\end{tabular}

\section{Monte Carlo estimators for the coupled system parameters $k_{i j}$ and $l_{i j}$}

$k_{i j}$ is defined as the average number of fission neutrons produced in the reactor $i$ by a neutron born in the reactor $j$. Implicit Monte Carlo estimators for these quantities were implemented in several codes, in which the (spatially discretized) Fission Matrix is scored (Carney et al., 2012). In this work, simple analog Monte Carlo estimators were preferred. This simplifies the implementation of the required adjoint-weighted calculation for the effective lives $\left(l_{i j}\right)$ and the sensitivity coefficients (see next section). In particular, the calculation of $k_{i j}$ involves the scoring of $s_{j}$ and $p_{i j}$. The first quantity represents the total weight of the source neutrons from the reactor $j$ in the present generation. This quantity is scored at the beginning of each Monte Carlo active cycle, checking the initial position of the source particles forming the whole neutron population:

$$
s_{j}=\sum_{n \in j} w_{n}
$$

$w_{n}$ represents the weight of the generic particle $n$. In case of analog Monte Carlo transport, all 
the particles have the same weight.

Moreover, a label is attached to each particle, to save the ID of the reactor $j$ of origin. A score $w_{n}$ is added to the buffer $p_{i j}$ every time a new fission neutron is produced in $i$ from a neutron labeled $j$ :

$$
p_{i j}=\sum_{n \in(i \leftarrow j)} w_{n}
$$

If $N$ is the total number of reactors in the system, $N x N p_{i j}$ buffers need to be allocated.

Estimates of the expected value for $k_{i j}$ are produced at the end of each cycle as the ratios of the $p_{i j}$ and $s_{j}$ scores:

$$
k_{i j} \sim \kappa_{\imath \jmath}=\frac{E\left[p_{i j}\right]}{E\left[s_{j}\right]}
$$

Statistical errors estimates for $k_{i j}$ and the other quantities presented in the following are obtained by comparing the results obtained from different cycles, as common in Monte Carlo codes:

$$
\operatorname{Var}\left(k_{i j}\right) \sim E\left[\left(k_{i j}\right)^{2}\right]-\left(E\left[k_{i j}\right]\right)^{2}
$$

$E\left[\left(k_{i j}\right)^{2}\right]$ represents the average of the squared $k_{i j}$ estimates over a large number of cycles.

$l_{i j}$ represents the effective prompt lifetime associated to the quantity $k_{i j}$. It can be view as the average lifetime of the neutrons born in $j$, producing a fission in $i$, weighted with the importance of the neutron. In cite (Laureau et al, 2015) the temporal aspect is introduced in the fission matrix approach by calculating the simple average time (i.e., not adjoint weighted) required by a neutron from $j$ to produce a fission in $i$. In this work, the importance weighting was introduced adopting the Iterated Fission Probability (IFP) capabilities available in SERPENT (Leppänen et al., 2014):

$$
l_{i j}=\frac{\sum_{n \in(j \leftarrow i)} w_{n} l_{n} I_{n}^{g}}{\sum_{n \in(j \leftarrow i)} w_{n} I_{n}^{g}}
$$

where the sums are performed over all the neutrons in the population labeled with an origin in the reactor $j$ and producing a fission in $i . w_{n}$ is the weight of the neutron $n$ and $l_{n}$ is the associated lifetime. The Iterated Fission Probability approach estimates the importance of a particle through its contribution to the asymptotic neutron population. In particular, the expected importance of the neutron $\left(I_{n}{ }^{(g)}\right)$ is evaluated as the sum of the weights $w_{k}$ of the descendants $k$ of the neutron $n$, produced after $g$ generations, normalized to the initial weight $w_{n}$ :

$$
I_{n}^{(g)} \sim I_{n}^{(g)} \propto \frac{1}{w_{n}} \sum_{k \in d_{n}^{(g)}} w_{k}
$$

The sum is performed over the set $d_{n}^{(g)}$ of the descendants.

Following the previous IFP implementation in SERPENT, adjoint weighted $l_{i j}$ estimators are scored on each cycle computing the average lifetime of the $g^{\text {th }}$ ancestors that originated in $j$ and produced a fission in $i$, over the present neutron population. This requires the additional 
propagation of the labels indicating the origin reactor $j$ from each particle to the descendant. (see Leppänen et al., 2014, for the details of the SERPENT IFP implementation).

\section{Monte Carlo calculation of sensitivity coefficients for $k_{i j}, l_{i j}$}

In this work, the collision history approach to Generalized Perturbation Theory (GPT) calculation previously implemented in SERPENT (Aufiero et al., 2015) was extended and adopted to estimate sensitivity coefficients involving $k_{i j}$ and $l_{i j}$ as response functions. The sensitivity coefficient $S_{x}{ }_{i j}$ is defined as the relative change in $k_{i j}$ the due to a perturbation in the generic parameter $x$. It represents the change in the probability of a neutron originated in $j$ to produce a fission in $i$. The formulation introduced by Avery allows the fission rates in different reactors $\left(S_{1}, S_{2} \ldots S_{N}\right)$ to be derived from the matrix of the $k_{i j}$ parameters. For this reason, the effects of perturbations in the system on $k_{i j}$ are of particular interest in predicting and understanding the behavior of power distribution in coupled reactors.

The present investigation was focused on the evaluation of sensitivities involving perturbations in material compositions. This GPT approach estimates the sensitivity of a generalized response $R$ to the atomic density of an isotope $x$ from the correlation between the Monte Carlo scores for $R$ and the accepted/rejected neutron collisions on $x$. In case of homogeneous problems (i.e., criticality source simulations), $R$ is required to be in the form of the ratio of two quantities:

$$
R=\frac{E\left[e_{1}\right]}{E\left[e_{2}\right]}
$$

where $E\left[e_{1}\right]$ and $E\left[e_{1}\right]$ are the expected value for the Monte Carlo estimators related to the generic quantities $e_{1}$ and $e_{2}$. These estimators are usually obtained from the sum of analog or implicit scores over the neutron population, at each generation.

In analog Monte Carlo transport, changes in the considered parameters (e.g., reaction cross sections or material densities) lead to different paths of the particles, with respect to the reference, unperturbed system. The collision history-based approach to GPT calculations adopts a particle weight perturbation technique. This way, both in the perturbed and unperturbed systems the neutron population undergoes the same event chains. Nonetheless, the effect of parameters perturbations is accounted for by changing the weights of the particles. In case of first-order analysis, the weight perturbation of a particle is related to number of the past collision events involving the perturbed parameter $x$.

This weight perturbation approach allows the derivatives of the generic quantities $e_{1}$ and $e_{2}$ to be estimated from the correlation between the particle collision events and the contribution of each particle to the scores for $E\left[e_{1}\right]$ and $E\left[e_{2}\right]$.

Usually, $R$ is defined as the ratio of the integrals of two linear functions of the forward flux:

$$
R=\frac{\left\langle\Sigma_{1}, \varphi\right\rangle}{\left\langle\Sigma_{2}, \varphi\right\rangle}
$$

or bi-linear functions of the forward and adjoint flux:

$$
R=\frac{\left\langle\varphi^{*}, \Sigma_{1} \varphi\right\rangle}{\left\langle\varphi^{*}, \Sigma_{2} \varphi\right\rangle}
$$


In case of $R=k_{i j}$, the Monte Carlo estimator is implemented as the ratio of $s_{j}$ and $p_{i j}$ (see previous section).

The sensitivity coefficient $\boldsymbol{S}_{x}{ }^{k} i j$ is obtained as:

$$
S_{x}^{k i j} \sim S_{x}^{k \imath \jmath}=\frac{\operatorname{COV}\left[p_{i j}, \sum^{\text {history }}\left(A C C_{x}-R E J_{x}\right)\right]}{E\left[p_{i j}\right]}-\frac{\operatorname{COV}\left[s_{j}, \sum^{\text {history }}\left(A C C_{x}-R E J_{x}\right)\right]}{E\left[s_{j}\right]}
$$

Where $\operatorname{COV}\left[p_{i j}, \operatorname{SUM}\left(A C C_{x_{-}} R E J_{x}\right)\right]$ and $\operatorname{COV}\left[p_{i j}, S U M\left(A C C_{x_{-}} R E J_{x}\right)\right]$ are the correlations between the scores of the Monte Carlo estimators for $p_{i j} s_{j}$, and the accepted/rejected collisions on the isotope $\boldsymbol{x}$, calculated over the whole neutron population. The sum is performed over the collision history buffer of each particle, to correctly take into account the effect of the perturbation on the fission source distribution (see Aufiero et al. 2015) $E\left[p_{i j}\right] E\left[s_{j}\right]$ are the expected value for $p_{i j} s_{j}$. Monte Carlo estimators for these quantities were presented above.

A similar approach has been adopted to calculate sensitivity coefficients for $l_{i j}$. In this case, the denominator term of the response function is the adjoint-weighted sum of the weights of the neutrons originated in $j$, that produce a fission in $i$. The numerator term is the adjointweighted sum of the neutron lifetimes involved in this process. The calculation of sensitivities for the power distribution $\left(S_{1} / S_{2}\right)$ makes use of the available GPT capabilities in the extended SERPENT version. $\mathrm{S}_{1} / \mathrm{S}_{2}$ is defined as:

$$
\frac{S_{1}}{S_{2}}=\frac{\left\langle\Sigma_{f 1}, \varphi\right\rangle}{\left\langle\Sigma_{f 2}, \varphi\right\rangle}
$$

The spatial integrations are performed over the two reactors.

The current collision history-based implementation produces unbiased estimates for the indirect components of the effect of a perturbation (i.e., effects due to the modification of the flux $\varphi$ ):

$$
\frac{\left\langle\Sigma_{f 1}, \frac{\partial \varphi}{\frac{\partial x}{x}}\right|}{\left\langle\Sigma_{f 1}, \varphi\right\rangle}-\frac{\left\langle\Sigma_{f 1}, \frac{\partial \varphi}{\frac{\partial x}{x}}\right|}{\left\langle\Sigma_{f 1}, \varphi\right\rangle}
$$

The direct component of the perturbation:

$$
\frac{\left\langle\frac{\partial \Sigma_{f 1}}{\partial \frac{x}{x}}, \varphi\right|}{\left\langle\sum_{f 1}, \varphi\right\rangle}-\frac{\left\langle\frac{\partial \Sigma_{f 2}}{\partial \frac{x}{x}}, \varphi\right|}{\left\langle\sum_{f 2}, \varphi\right\rangle}
$$

is estimated separately (when present) via collisional detectors in an independent standard SERPENT calculation.

\section{Verification and Comparison against Deterministic Methodology}

In order to verify the accuracy of the sensitivity Monte Carlo estimators presented above, a simple case study has been selected to test the GPT estimates against direct perturbation and ERANOS (Rimpault et al., 2002) reference results. Continuous-energy Monte Carlo results 
were obtained with Serpent, adopting the ENDF/B-VII nuclear data library. The case study consists in the spherical "coupled" system represented in Fig. 3.

Figure 3. Simple model for verification calculations.

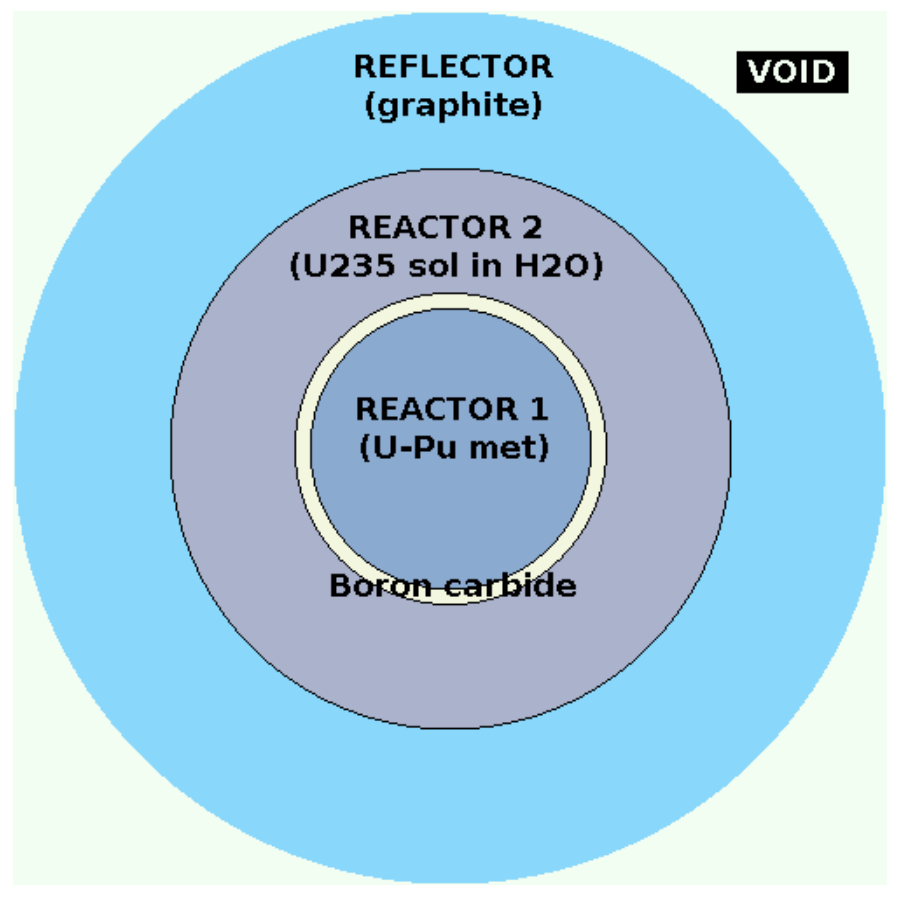

The central region ("fast reactor") consists in a $9 \mathrm{~cm}$ sphere of a (30\%-70\%) mixture of metallic ${ }^{239} \mathrm{Pu}$ and ${ }^{238} \mathrm{U}$. The "thermal reactor" is made of a $9 \mathrm{~cm}$ thick spherical region of a water solution of ${ }^{235} \mathrm{U}(5.9 \mathrm{w} \%)$. The two regions are separated by a $1-\mathrm{cm}$ thick boron carbide filter, to prevent the thermal neutrons from inducing fissions in the fast reactor. A graphite reflector surrounds the whole system. Figure 4 shows the radial neutron flux distribution in the systems, divided in three energy groups: $\mathrm{E}>0.1 \mathrm{MeV}, 0.1 \mathrm{keV}<\mathrm{E}<0.1 \mathrm{MeV}$ and $\mathrm{E}<$ $0.1 \mathrm{keV}$.

Figure 4. Radial neutron flux distribution in the simple model (three energy groups). 


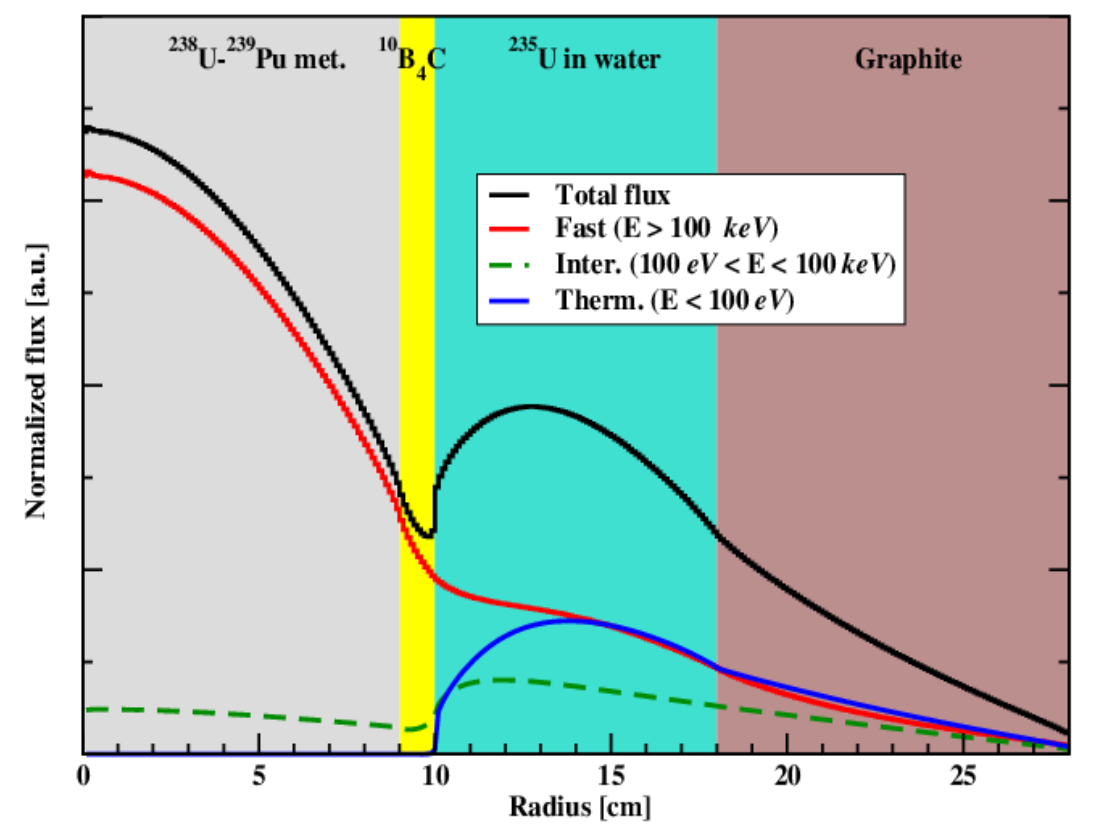

The curves were obtained via standard collisional detector mesh in SERPENT, and show how the fast flux is highly peaked in the central region, whereas the low-energy flux is peaked in the center of the thermal region. Figure 5 shows the fission rate weighted spectra in the two reactors.

Figure 5. Fission rate weighted spectra in the two reactors of the simple model.

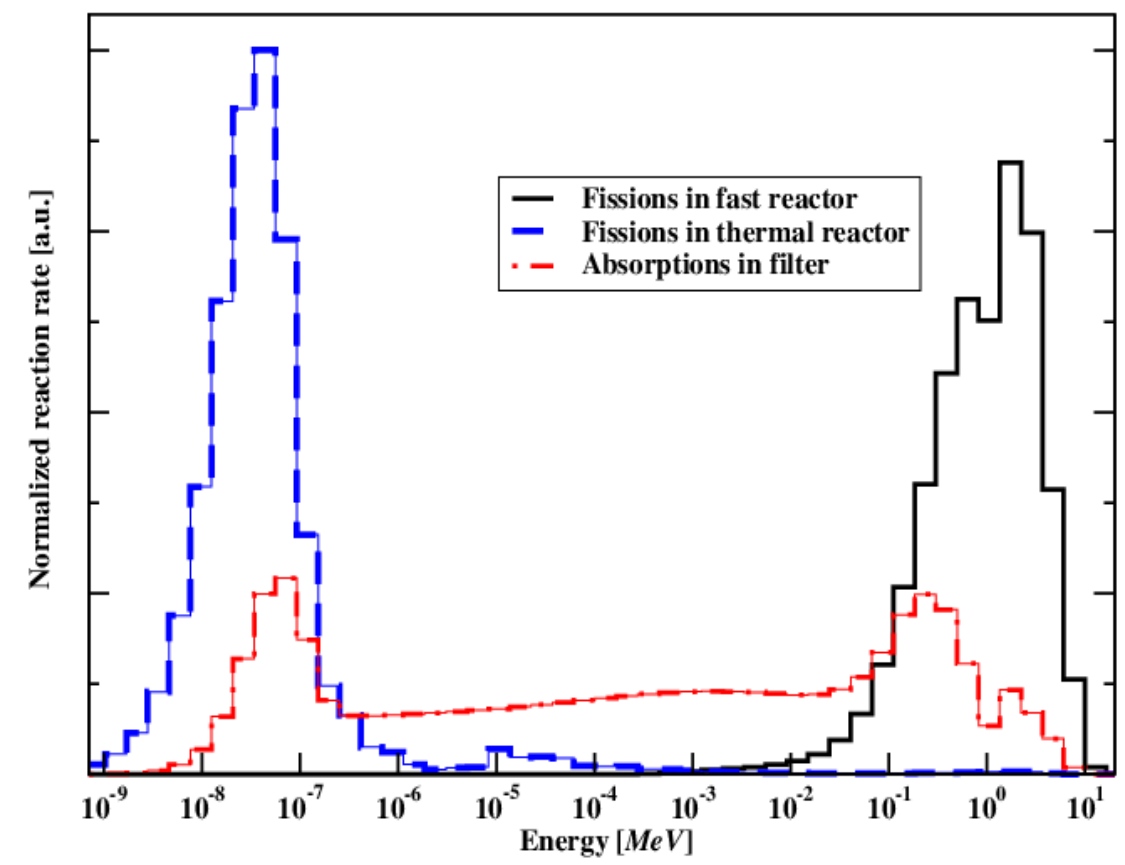

The reference values for the Avery's coupling coefficients obtained with the Monte Carlo estimators presented above are reported in Table I, and compared to ERANOS deterministic calculations. The subscripts 1 and 2 refer to the fast and thermal reactors, respectively. 


\begin{tabular}{|c|c|c|c|c|}
\hline \multirow[b]{2}{*}{ Parameter } & \multicolumn{2}{|c|}{ SERPENT } & \multirow[b]{2}{*}{ ERANOS } & \multirow[b]{2}{*}{$\begin{array}{l}\text { Rel. } \\
\text { diff. }\end{array}$} \\
\hline & Value & $\begin{array}{c}\text { Uncertainty } \\
\text { (STD) }\end{array}$ & & \\
\hline $\begin{array}{l}\boldsymbol{k}_{\text {eff }} \text { all } \\
\text { system }\end{array}$ & 1.00074 & $\pm 10 \mathrm{pcm}(\mathrm{abs})$ & 1.00031 & $0.04 \%$ \\
\hline $\begin{array}{l}\text { eff all } \\
\text { system }\end{array}$ & $18.37 \mu \mathrm{s}$ & $\pm 0.1 \%(\mathrm{rel})$ & $17.69 \mu \mathrm{s}$ & $3.8 \%$ \\
\hline$k_{11}$ & 0.80023 & $\pm 25 \mathrm{pcm}(\mathrm{abs})$ & 0.82576 & $-3.1 \%$ \\
\hline$k_{12}$ & 0.07115 & \pm 5 pcm (abs) & 0.078126 & $-8.9 \%$ \\
\hline$k_{21}$ & 0.55199 & $\pm 20 \mathrm{pcm}(\mathrm{abs})$ & 0.56959 & $-3.1 \%$ \\
\hline$k_{22}$ & 0.80418 & $\pm 13 \mathrm{pcm}(\mathrm{abs})$ & 0.80254 & $0.2 \%$ \\
\hline$l_{11}$ & $9.11 \mathrm{~ns}$ & $\pm 0.2 \%(\mathrm{rel})$ & $10.41 \mathrm{~ns}$ & $-12.5 \%$ \\
\hline$l_{12}$ & $20.97 \mathrm{~ns}$ & $\pm 0.3 \%$ (rel) & $20.21 \mathrm{~ns}$ & $3.8 \%$ \\
\hline$l_{21}$ & $35.86 \mu \mathrm{s}$ & $\pm 0.3 \%$ (rel) & $35.17 \mu \mathrm{s}$ & $2.0 \%$ \\
\hline$l_{22}$ & $38.69 \mu \mathrm{s}$ & $\pm 0.1 \%(\mathrm{rel})$ & $37.82 \mu \mathrm{s}$ & $2.3 \%$ \\
\hline
\end{tabular}

Table I. Comparison of coupling coefficients and neutron prompt lifetimes between SERPENT and ERANOS.

The results of table I show that both "reactors" present separately an effective multiplication factor around 0.8. The neutronic coupling between the two regions is strongly asymmetric: the values of $k_{12}$ and $k_{21}$ confirm the filtering effect of the boron carbide layer in limiting the probability for neutrons produced in the thermal reactor to produce fission in the central region. On the other hand, neutrons produced in the fast reactor present a large probability to cross the filter region and induce a fission in the thermal reactor

SERPENT results appear in fairly good agreement with ERANOS values, confirming the consistency of the implemented estimators. For the verification of the sensitivity/perturbation calculations against direct perturbation results, the atomic density of $\mathrm{H}$ in the thermal reactor and ${ }^{10} \mathrm{~B}$ in the filter region were changed by $5 \%$. Independent Monte Carlo criticality source simulations were performed, adopting $10 \times 10^{9}$ for both the reference and the perturbed cases. In this way, the sensitivities of $k_{i j}, l_{i j}$ and S1/S2 were accurately obtained via numerical differentiation, and compared to the GPT estimates.

The comparisons are presented in table II and table III. Results are expressed as sensitivity coefficients (relative values of $(\delta R / R) /(d \sigma / \sigma))$, where $R$ is the integral parameter and $\sigma$ the variable). The non-linear errors introduced by the finite perturbation $(5 \%)$ in the direct perturbation approach were neglected. The statistical uncertainties in the relative difference between GPT and reference results accounts for sum of the variance of the two methods.

\section{Sensitivity Studies}

The sensitivity coefficient analysis allows a detailed understanding of the coupling phenomena. Some examples will be given in this paragraph, related to the model coupled reactor of figure 3. As shown above in figure 4, the flux radial distributions by energy band have been calculated as well as the fission rate weighted spectra in the two fissile regions, and they provide the basis for understanding the physics of the sensitivity analysis. In fact a 
number of perturbations were analyzed, e.g. the variation of the hydrogen density in Reactor 2 or of the boron content in the filter region (see figure 3 ). ${ }^{238} \mathrm{U}$ individual cross sections have also been considered.

\begin{tabular}{|c|c|c|c|c|}
\hline Parameter & GPT & $\begin{array}{c}\text { Direct } \\
\text { pert. }\end{array}$ & $\begin{array}{c}\text { Rel. diff. } \\
(\%)\end{array}$ & $\begin{array}{c}\text { Uncertainty (rel. STD } \\
\%)\end{array}$ \\
\hline $\boldsymbol{k}_{\boldsymbol{1 1}}$ & -0.031 & -0.028 & -8.6 & 4.0 \\
\hline $\boldsymbol{k}_{\boldsymbol{1 2}}$ & 0.341 & 0.337 & -1.0 & 0.5 \\
\hline $\boldsymbol{k}_{\boldsymbol{2} 1}$ & -0.559 & -0.551 & -1.5 & 0.7 \\
\hline $\boldsymbol{k}_{\boldsymbol{2} 2}$ & 0.453 & 0.453 & 0.0 & 0.2 \\
\hline $\boldsymbol{l}_{\boldsymbol{1 1}}$ & -0.041 & -0.050 & 22.9 & 13.4 \\
\hline $\boldsymbol{l}_{\boldsymbol{1 2}}$ & -0.367 & -0.367 & -0.1 & 2.8 \\
\hline $\boldsymbol{l}_{\boldsymbol{2}}$ & -0.268 & -0.252 & -6.0 & 4.9 \\
\hline $\boldsymbol{l}_{\boldsymbol{2} 2}$ & -0.255 & -0.250 & -2.3 & 2.4 \\
\hline
\end{tabular}

Table II. Sensitivity coefficients to hydrogen density via perturbation theory and direct calculations.

In fact, as an example, a higher $\mathrm{H}$ density in the thermal spectrum region, increases, as expected, the $k_{22}$ as indicated in table II. It also increases the coupling effect represented by $k_{21}$, since fast neutrons coming from the fast spectrum region will have more probability of slowing down to thermal energies where the ${ }^{235} \mathrm{U}$ fission cross section increases. On the contrary, the well thermalized neutrons in region 2 will have more probability to be captured (because of higher capture cross section) by the B-10 in the filter and, consequently, the coupling coefficient $k_{12}$ will decrease.

\begin{tabular}{|c|c|c|c|c|}
\hline Parameter & GPT & $\begin{array}{c}\text { Direct } \\
\text { pert. }\end{array}$ & $\begin{array}{c}\text { Rel. diff. } \\
(\%)\end{array}$ & $\begin{array}{c}\text { Uncertainty (rel. STD } \\
\%)\end{array}$ \\
\hline $\boldsymbol{k}_{\boldsymbol{1 1}}$ & 0.027 & -0.027 & $3.0 \%$ & $0.7 \%$ \\
\hline $\boldsymbol{k}_{\boldsymbol{2} 1}$ & -0.197 & -0.198 & $0.3 \%$ & $0.1 \%$ \\
\hline $\boldsymbol{k}_{\boldsymbol{1 2}}$ & -0.221 & -0.220 & $-0.5 \%$ & $0.2 \%$ \\
\hline $\boldsymbol{k}_{\mathbf{2}}$ & -0.023 & -0.023 & $-0.2 \%$ & $0.4 \%$ \\
\hline $\boldsymbol{l}_{\boldsymbol{1 1}}$ & -0.082 & -0.081 & $-1.4 \%$ & $1.5 \%$ \\
\hline $\boldsymbol{l}_{\boldsymbol{2} 1}$ & 0.013 & 0.009 & $-29.6 \%$ & $9.4 \%$ \\
\hline $\boldsymbol{l}_{\boldsymbol{1} 2}$ & -0.342 & -0.334 & $-2.5 \%$ & $0.7 \%$ \\
\hline $\boldsymbol{l}_{\boldsymbol{2} 2}$ & 0.014 & 0.007 & $-49.3 \%$ & $45.2 \%$ \\
\hline
\end{tabular}

Table III. Sensitivity to ${ }^{10} \mathrm{~B}$ density via perturbation theory and direct calculations

The decrease of the S1/S2 ratio is also expected, in view of the enhanced role of the thermal region in terms of power share, which is shown in fig. 5.

The effect of the variation of the boron content in the filter has smaller effects as shown by the sensitivity coefficients of table III (e.g. the increase of $5 \%$ of the boron content induces only $\sim 1 \%$ decrease of the coupling coefficients $k_{12}$ or $k_{21}$ ). The effect on the power share is even smaller.

As for the coupling coefficient $k_{21}$, which accounts for neutrons born in the fast neutron region and giving fission in the thermal spectrum region, the sensitivity profiles of Fig. 6 
indicate that the $k_{21}$ decreases both as a consequence of an increase of the low energy absorptions of $\mathrm{H}$ (in the "thermal" zone) and of an increase of the filter ${ }^{10} \mathrm{~B}$ total cross section at high energy. On the contrary, an increase of the $\mathrm{H}$ elastic cross section increases the thermalisation of the fast neutrons, increasing their contributions to the fission in the thermal spectrum zone.

Figure 6. Extended SERPENT-2 GPT calculations of $k_{21}$ sensitivity to Hydrogen and Boron cross sections.

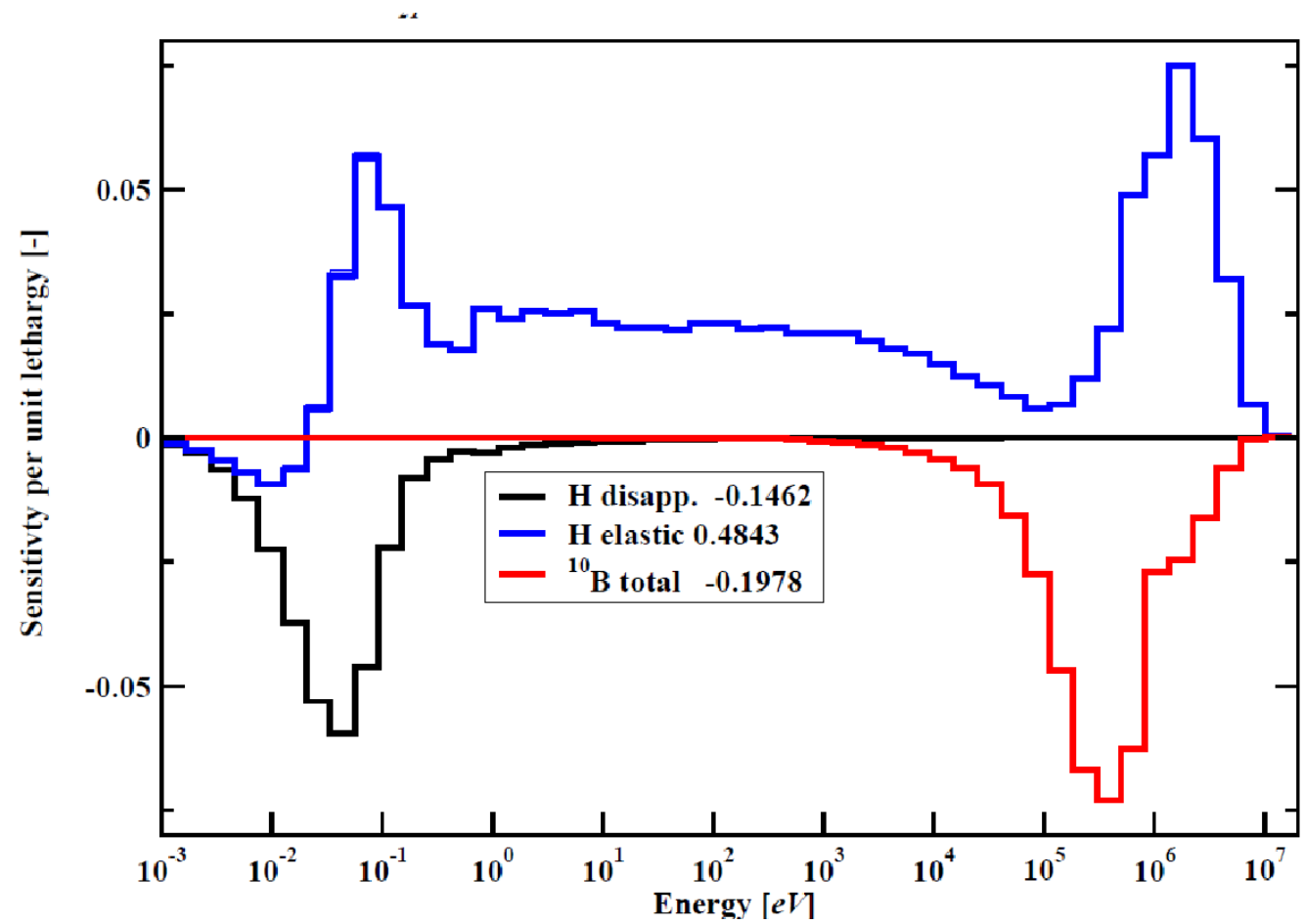

As an example of individual cross sections impact on the coupling parameters, Fig. 7 indicates that any increase of the inelastic cross section of ${ }^{238} U$ at very high energy $(>2-3$ $\mathrm{MeV})$ brings neutrons to the high flux region $(\sim 100 \mathrm{keV}-1 \mathrm{MeV})$, increasing the probability of leakage towards the thermal region, i.e. the $k_{21}$ increases. On the contrary, an increase of the same cross section at energies below $\sim 1 \mathrm{MeV}$, will reduce the number of neutrons able to reach the thermal spectrum region, i.e. the $\mathrm{k}_{21}$ decreases.

In the same figure the effects of the ${ }^{238} \mathrm{U}$ fission spectrum are also shown. They indicate an opposite trend with respect to the inelastic cross section variation: in fact e.g. an increase of the fission spectrum in the energy region below $\sim 1 \mathrm{MeV}$ means "useful (for $k_{12}$ ) neutron production", whereas an increase of the inelastic cross section in that same energy region means "useful (for $k_{12}$ ) neutron disappearance". Lastly, the total effects accounts also for the total absorption and the elastic scattering variations, that further contribute e.g. to the "useful (for $\boldsymbol{k}_{12}$ ) neutron disappearance" below $\sim 1-2 \mathrm{MeV}$.

Figure 7. Extended SERPENT-2 GPT calculations of $k_{21}$ sensitivity to ${ }^{238} \mathrm{U}$ cross sections. 


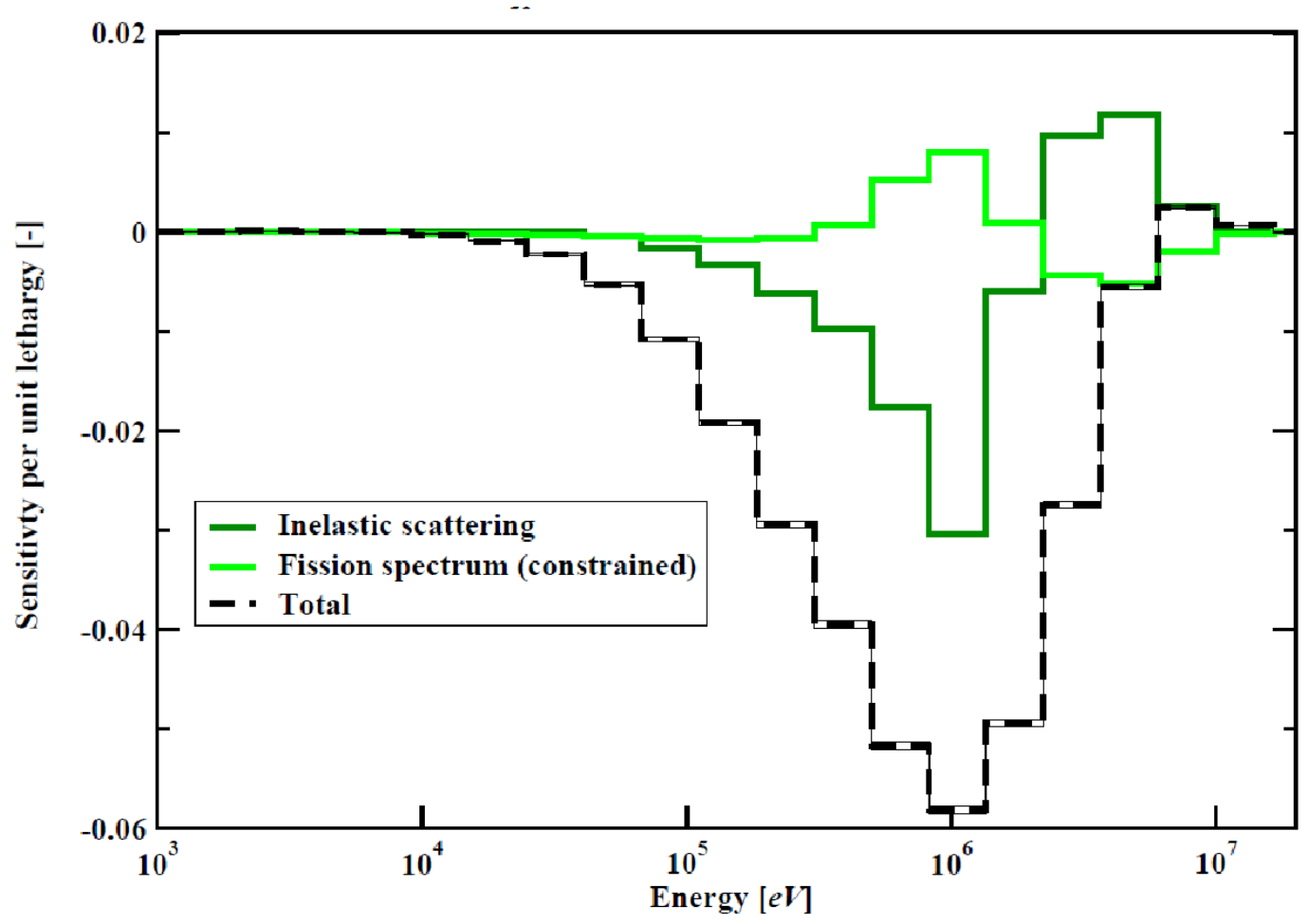

Finally, Fig. 8 shows that increasing the boron absorption in the filter zone has the effect of decreasing the ratio $R_{I}=$ (integral flux in the range $100 \mathrm{eV}<\mathrm{E}<100 \mathrm{keV}$ )/ (total flux) due to the intermediate born absorptions and of decreasing even more the ratio $R_{T}=$ (integral flux below $100 \mathrm{eV}$ )/ (total flux) due to the very high absorption rate of neutron born in that energy range. Such a type of indications can help to optimize e.g. the filter composition for specific objectives.

These sensitivity analyses can be of interest also in cases of large cores with significant coupling effects, as indicated previously. The modified SERPENT code will then be a particularly useful tool of analysis.

\section{Conclusions}

Coupled reactors and the coupling features of large or heterogeneous core reactors can be investigated with the Avery's theory that allows a physics understanding of the main features of these systems. However, the complex geometries that are often encountered in association with coupled reactors, require a detailed geometry description that can be easily provided by modern Monte Carlo (MC) codes. This implies a MC calculation of the coupling parameters defined by Avery and of the sensitivity coefficients that allow further detailed physics analysis. The results presented in this paper show that the MC code SERPENT has been successfully modified to meet the required capabilities.

Future work will address the analysis of the key issue of power shape potential tiltiness in coupled systems, using the relation between Boltzmann equation eigenvalue separation and Avery coupling coefficients. 
Figure 8. Extended SERPENT-2 GPT $\mathrm{B}_{4} \mathrm{C}$ filter zone. Spectral indexes sensitivity to ${ }^{10} \mathrm{~B}$ disappearance $((n, \alpha)+(n, \gamma))$ cross sections.

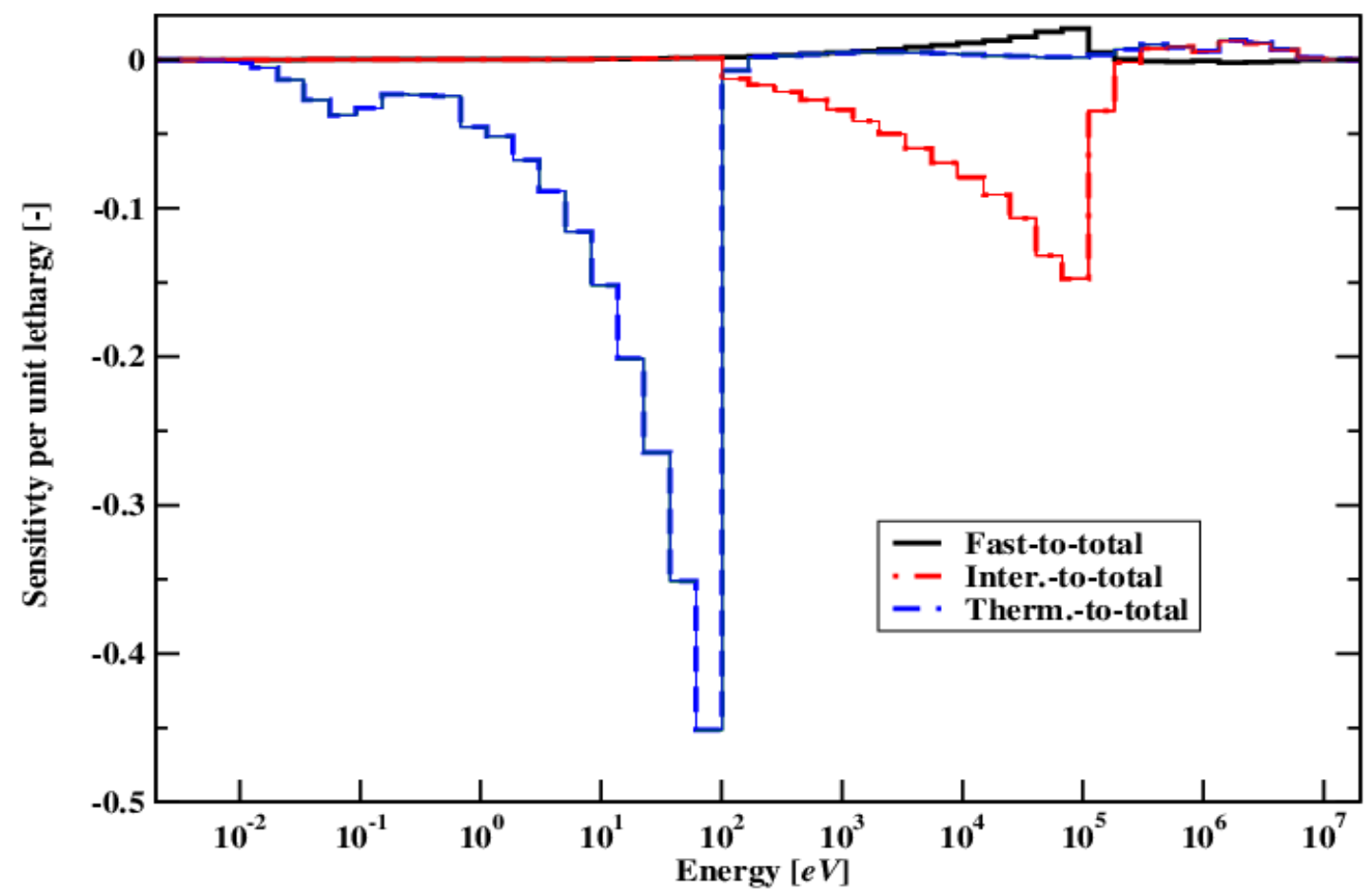

\section{Acknowledgments}

This submitted manuscript was authored by a contractor of the U.S. Government under DOE Contract No. DE-AC07-05ID14517. Accordingly, the U.S. Government retains and the publisher, by accepting the article for publication, acknowledges that the U.S. Government retains a nonexclusive, paid-up, irrevocable, world-wide license to publish or reproduce the published form of this manuscript, or allow others to do so, for U.S. Government purposes. 


\section{References}

Abramov, 2001 B.D. Abramov "Modifications to the Theory of Coupled Reactors", Atomic Energy, Vol. 90, No. 5, 2001

Aufiero et al. 2015 M. Aufiero et al., "A Collision History-Based Approach To Sensitivity/Perturbation Calculations In The Continuous Energy Monte Carlo Code SERPENT", Annals of Nuclear Energy 85 (2015) 245-258.

Avery, 1958 R. L. Avery “Theory of Coupled Reactors” Proc. $2^{\text {nd }}$ Int. Conf. Peaceful Uses of At. Energy, Vol. 12, 182 (1958)

Baldwin, 1959 G. C. Baldwin, "Kinetics of a Reactor Composed of Two Loosely Coupled Cores", Nucl. Sci. Eng. 6, 320 (1959)

Bustraan et al. 1970 M. Bustraan et al., "STEK, the Fast-Thermal Coupled Facility of RCN at Petten", RCN-122 (1970)

Carney et al., 2012 Carney, S.E., Brown, F.B., Kiedrowski, B.C., Martin, W.R., 2012. Fission Matrix Capability for MCNP Monte Carlo. LA-UR-12-24533, Los Alamos National Laboratory.

Komata, 1969 M. Komata, “On the Derivation of Avery's Coupled Reactor Kinetics Equations”, Nucl. Sci. Eng. Vol. 38, 193 (1969)

Kukharchuk et al., 2000 O. F. Kukharchuk et al., "Coupled Fast-Thermal System : Theory and Experiments », Proc. Int. Conf. PHYSOR 2000

Laureau et al., 2015 A. Laureau et al. , "Coupled Neutronics And Thermal-Hydraulics Transient Calculations Based On A Fission Matrix Approach: Application To The Molten Salt Fast Reactor", ANS MC2015 - Joint International Conference on Mathematics and Computation (M\&C), Supercomputing in Nuclear Applications (SNA) and the Monte Carlo (MC) Method, Nashville, Tennessee, April 19-23, 2015

Leppänen et al., 2014 J. 15- $\quad$ Leppänen et al. "Calculation Of Effective Point Kinetics Parameters In The SERPENT 2 Monte Carlo Code", Annals of Nuclear Energy, 65, (2014) 272-279.

Meister et al., 1964 H. Meister et al., "The Karlsruhe Fast-Thermal Argonaut Reactor Concept" KFK-217 (1964)

Palmiotti, Salvatores et al., 2016 G. Palmiotti, M. Salvatores et al., "Spatial effects of neutron coupling/decoupling in different types of reactors and impact on neutronics performances", to be submitted to Annals of Nuclear Energy

Rimpault, et al., 2002 G. Rimpault, et al., "The ERANOS Code and Data System for Fast Reactor Neutronic Analyses", Proc. Physor 2002 Conference, Seoul (Korea), October 2002

Seale, 1964 R.L. Seale “Coupled Core Reactors”, LAMS-2967 (1964) 
Sen et al., 2016 S. Sen et al., "A Versatile Innovative Thermal-Fast Coupled Test Reactor", Proc. Physor 2016 Conference, Sun Valley, Idaho (USA), May 20166

Toppel, 1957 B. J. Toppel "The Coupled Aspects of a Fast-Thermal Critical: ZPR-V", ANL-5775 (1957)

Varaine et al., 2012 Frédéric Varaine et. al, "Pre-conceptual design study of ASTRID core”, Proceedings of ICAPP '12, Chicago, USA, June 24-28, 2012 